\title{
Analysis of Hadron Interactions Detected in Balloon- borne Emulsion Chamber by Automated Emulsion Read-out System
}

\author{
Misaki Morishita On behalf of GRAINE Collaboration \\ Nagoya University \\ Furo-cho, Chikusa-ku, Nagoya, 464-8602, Japan \\ E-mail: morishitaeflab.phys.nagoya-u.ac.jp
}

Nuclear emulsion is a tracking detector which has very high spacial resolusion of about 1 micron and records all charged particles passed through. And it is realtively easy to build large scale detector using emulsion. The purpose of GRAINE (Gamma-Ray Astro-Imager with Nuclear Emulsion) project is precise observation of cosmic gamma-rays by using a balloonborne emulsion detector. All phenomena occurred in flight are recorded in emulsion and it is interesting to study not only gamma-ray events but also hadron interaction events induced by primary cosmic rays. Especially study of charmed particle production in primary cosmic rays interactions at high altitude is major interest of this article, since this information is important to validate prompt neutrino (atmospheric neutrino) flux as most of them are decay products of charmed particles. By using automated emulsion readout system HTS (Hyper Track Selector), it is possible to readout all tracks recorded in emulsion and analyze all hadron interactions to search for charmed particle decays.

This article describes a preliminary result of a test experiment using CERN accelerator beam. A method to estimate energy of primary cosmic rays by an automated analysis is checked in this test.

The 3rd International Symposium on "Quest for the Origin of Particles and the Universe" 


\section{Introduction}

Nuclear emulsion is a tracking detector which has very high spacial resolusion of about 1 micron and records all charged particles passed through. And it is realtively easy to build large scale detector using emulsion. High spacial resolution of emulsion is suitalbe to detect charmed particles through its decay topology, which occures in very short distance ( less than $1 \mathrm{~mm}$ ). In fact, charmed particle production was discovered in primary cosmic ray interactions recoreded in an emulsion chamber in 1971 by K.Niu et.al. [1]. It had been difficult to analyze all primary cosmic ray interactions in emulsion, since emulsion analysis had been rather time consuming in those days, and thus analysis was limited only on high energy part. Now by using automated emulsion readout system HTS ( Hyper Track Selector )[2] , it is possible to readout all tracks recorded in emulsion.

The purpose of GRAINE (Gamma-Ray Astro-Imager with Nuclear Emulsion) project is precise observation of cosmic gamma-ray by using a balloon-borne emulsion detector. [3] A test flight in Australia had successfully been done in May 2015 and a flight for scientific observation with emulsion telescope of $10 \mathrm{~m}^{2}$ aperture is being planned. Nuclear emulsion used in GRAINE has higher sensitivity compared to OPERA experiment and suitable for HTS readout. Since it records all phenomena occurred in flight in emulsion, it is interesting to study not only gamma-ray events but also hadron interaction events include by primary cosmic ray (e.g. proton, helium etc.), which occurred in this converter.

A study is charmed particle production due to primary comsic rays at high alititude is one of such interesting physics topic. This information is important to validate prompt neutrino ( atmospheric neutrino ) flux because most of them are decay products of charmed particles produced in primary cosmic ray interactions. In this article a preliminary result of a test experiment using CERN accelerator beam to validate a method to estimate energy of primary cosmic rays by an automated analysis, is report.

\section{Energy reconstruction by using Pseudo Rapidity Distribution}

Major component of primary cosmic rays are protons and charmed particle production cross section in proton interaction increases significantly around $100 \mathrm{GeV}$ [4]. Thus, a study of charmed particle production should be first concentrated for such interactions and, for this purpose, a systematic method to estimate primary particle species and its energies of is important. A method called Castagnoli's method [5] is suitable for emulsion because of its high special resolution. It estimates energy of primary particle by using pseudo rapidity distribution of charged secondary particles.

Fig.1 shows a three-dimensional picture of a hadron interaction found in the converter layer of GRAINE emulsion. Track segments observed in five emulsion plates are drawn in this figure. This interaction has one primary track, 41 secondary tracks and two evaporation tracks from the target nucleus. For a relativistic particle, its energy deposition $-\mathrm{dE} / \mathrm{dx}$ is correlated to its charge and PHvol information provided by HTS correlates with $-\mathrm{dE} / \mathrm{dx}$. Thus, one can estimate relativistic particle's 
charge using PHvol given in HTS track data. A primary track of this interaction had large PHvol value and thus it could not be a proton but should be a heavy nucleus.

Energy of this primary particle is estimated to by using pseudo rapidity distribution of 41 secondary tracks, which is shown in Fig.2. Assuming that secondary particles are emitted isotropic, central value of this pseudo rapidity distribution gives its primary particle energy to be $20 \mathrm{GeV} / \mathrm{A}$. By applying such analysis to all interactions reconstructed in the converter, hadronic interactions for charmed particle production study can be selected systematically.
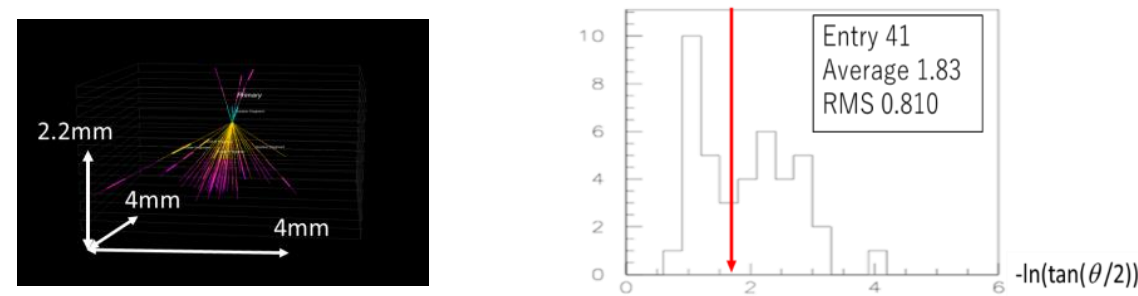

Fig.1 A three-dimensional picture of a hadron interaction observed in GRAINE

Fig.2 Pseudo rapidity distribution og the event described in the test

\section{Test Experiment using CERN Accelerator Beam}

\subsection{Purpose of a Test Experiment}

Hadron interactions recorded in GRAINE chamber is due to primary cosmic rays and their energy are not know a priori. Then it is rather bifficult to validate a method described in previous secton by using data obtained in GRAINE chamber only. Therefore a test experiment to collect interactions of know primary energy, i.e. accerelator beam, was performed. The method is exained by appying to those interactions to see if their energy is correctly reconstructed.

\subsection{Test Experiment at CERN}

A test experiment using CERN accelertor beam was done in Nov 2016 to validate the method described in previous section. Emulsion chambers used in this test have the same structure as of one unit of GRAINE converter layer. $400 \mathrm{GeV} / \mathrm{c}$ proton beam was exposed with a density of $1.8 \times 10^{4} / \mathrm{cm}^{2}$. An interaction density of $12 \pm 0.3$ (stat.) $/ \mathrm{cm}^{2}$ in one emulsion plate is expected from nuclear inteaction length of protons. All tracks recorded in emulsion are readout by HTS and hadronic interacions of beam particles are reconstructed using the same analysis method as used in GRAINE. Reconstruction efficiency and reconstructed beam energy are studied. 


\subsection{Comparison of Data with Simulation}

Pseudo rapidity $(\eta)$ distribution of charged secondary particles, emitted from hadronic interactions is compared with GEANT4 simulation. A result obtained with 14 events among 139 interaction candidates analyzed so far is shown in Fig. 3.

Mean value of $\eta$ is consistent with simulation, which 3.3 for $400 \mathrm{GeV}$ proton interaction. And the distribution of $\eta$ is reproduced rather well by simulation except for a discrepancy in forward region $(\eta>4.4)$. This discrepancy could be understood by considering HTS track recognition algorithm. A relative distance of tracks emitted in forward region could be very close and, in such case, HTS recognizes them as one track. This should be checked by implementing this HTS characteristics in simulation code or by using secondary tracks recognized in more downstream plates, where tracks in forward region are well separated.

\section{Summary}

A study of charmed particle production due to primary comsic rays at high alititude, which are recored in GRAINE chamber has an interesting physics, since this information is important to validate prompt neutrino ( atmospheric neutrino ) flux and charmed particle decay is a major source of such neutrinos.

As a first step for such an physics analysis, a test experiment using CERN accelerator beam was done to validate Castagnoli's method to estimate energy of primary cosmic rays, by an automated analysis based on HTS. A preliminary result on pseudo rapidity distribution using 14 hadron interactions analyzed so far is reported. There was a good agreement between data and simulation except for very forward region $(\eta>4.4)$. Further check is to be done to understand this discrepancy by understanding HTS track recognition algorithm and offline codes used in vertex reconstruction.

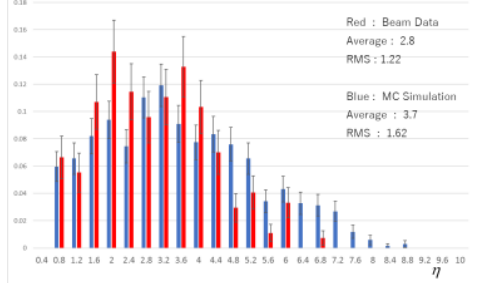

Fig.3 Pseudo rapidity distribution of 14 events obtained in test experiment

\section{References}

[1] S.Takahashi et.al PTEP, (DOI: 10.1093/ptep/ptw089)

[2] Kiyoshi Niu et al Prog. Theor. Hys. Vol. 46(1971), No.5.

[3] M.yoshimoto et al arXiv:1704.06814v1

[4] G. Altrarelli Nuclear Physiscs B vol.308(1988) 724-752

[5] N.A.Korotkova et al Physics of Atomic Nuclei vol.65(2002) 852-860 